\title{
Clinicopathological Significance of DLC-1 Expression in Cancer: a Meta-Analysis
}

\author{
Yan Jiang, Jian-Ming Li, Huai-Qing Luo*
}

\begin{abstract}
Background: Recent reports have shown that DLC-1 is widely expressed in normal tissues and is downregulated in a wide range of human tumors, suggesting it may act as a tumor suppressor gene. We conducted a meta-analysis to determine the correlation between DLC-1 expression and clinicopathological characteristics in cancers. Materials and Methods: A detailed literature search was made for relevant publications from PubMed, EMBASE, Cochrane library databases, Web of Science, CNKI. The methodological quality of the studies was also evaluated. Analyses of pooled data were performed and odds ratios (ORs) were calculated and summarized. Results: Final analysis was performed of 1,815 cancer patients from 19 eligible studies. We observed that DLC1 expression was significantly lower in cancers than in normal tissues. DLC-1 expression was not found to be associated with tumor differentiation status. However, DLC-1 expression was obviously lower in advance stage than in early-stage cancers and was more down-regulated in metastatic than non-metastatic cancers. Conclusions: The results of our meta-analysis suggested that DLC-1 expression is significantly lower in cancers than in normal tissues. Aberrant DLC-1 expression may play an important role in cancer genesis and metastasis.
\end{abstract}

Keywords: DLC-1 expression - metastasis - meta-analysis - cancer

Asian Pac J Cancer Prev, 16 (16), 7255-7260

\section{Introduction}

Distant metastasis is always occurred in advanced cancer and is proved to be the leading cause of cancer mortality, so it is a predominant issue to understand the molecular mechanisms that enables this phenomenon in cancer biology. Metastasis suppressor gene deregulation was certified to play a key role in metastasis and several new metastasis suppressor genes have been fond and studied in cancers. DLC-1, a member of the deleted-inliver cancer family of proteins which includes DLC-1, DLC2 and DLC3, was first identified as a tumor metastasis suppressor gene in hepatocellular carcinoma (Yuan et al., 1998). The human DLC-1 gene is localized on chromosome 8p21-22 and encodes a 1,091 amino acid protein that is highly homologous to the rat p122-RhoGAP, which is a GTPase-activating protein(GAP) for Rho family proteins (Homma and Emori, 1995). Rho family proteins play essential roles in regulating cytoskeletal organization, cell adhesion, and cellcycle progression (Etienne-Manneville and Hall, 2002; Moon and Zheng, 2003; Tcherkezian and Lamarche-Vane, 2007). It was determined that DLC-1 is widely expressed in normal tissues and is down-regulated in a wide range of tumor tissues.

Underexpression of DLC-1 was associated with either heterozygous deletions of the DLC-1 gene or hypermethylation of the gene promoter region (Guan et al., 2006; Seng et al., 2007; Guan et al., 2012). Recent studies have extensively investigated DLC-1 expression and function in many human cancers, including liver, breast, lung, ovarian, kidney, colon, stomach, prostate, nasopharynx, Gallbladder and so on (Guan et al., 2006; Peng et al., 2006; Zhang et al., 2009; Hua et al., 2010; Yufei et al., 2010; Fan and Shi, 2011; Yun et al., 2011; Fang et al., 2012; Quanrui and Zhimei, 2012; Peng et al., 2013; Ren et al., 2013; Yang et al., 2013a; Yang et al., 2013b; Feng et al., 2014; Mingrui et al., 2014; Qin et al., 2014; Yufei et al., 2014; Yujie et al., 2014; Zhefeng et al., 2015). Wang Y reported that DLC-1 is an important regulator of TGF- $\beta$ responses and DLC- 1 overexpression suppressed bone metastasis of breast cancer (Wang et al., 2014). Ren reported that the expression of DLC-1 was closely related with the metastasis and invasion of ovarian carcinoma (Ren et al., 2013). However, controversies still exist due to the limited number of patients in individual studies. In addition, the association between DLC-1 expression and clinical significance has not been thoroughly investigated. In this study, we pooled and analyzed the published clinical investigations regarding the effect of DLC-1 on cancer patients.

\section{Materials and Methods}

\section{Search strategy and selection criteria}

The following electronic databases were searched for relevant articles without any language restrictions: 
PubMed (data from 1966 to March 2015), Excerpta Medica dataBASE (EMBASE) (data from 1980 to March 2015), Cochrane library databases (up to March 2015), Web of Science (1945-2015) and China National Knowledge Infrastructure databases (CNKI). The search items are as follows: "cancer or tumor or neoplasm or carcinoma," "expression," "DLC-1 or DLC-1 or deletedin-liver cancer 1," "prognosis or prognostic or outcome."

The criteria that an eligible study had to meet were as follows: (1) DLC-1 expression evaluated in primary cancer tissues, (2) researchers revealed the relationship between DLC-1 expression and cancer clinicopathological parameters and prognosis, and (3) studies provided sufficient information to estimate odds ratio (OR) and 95 $\%$ confidence interval (CI) about prognosis . The exclusion criteria included the following: (1) letters, reviews, case reports, conference abstracts, editorials, expert opinion and (2) all publications regarding in cell lines and animal models.

\section{Data extraction and methodological assessment}

Two authors (Jiang and Li) independently reviewed and extracted data from eligible studies. Disagreements were resolved by discussion with a third reviewer (Luo). The following information was recorded for each study: the first author name, year of publication, sample source, number of cases, clinicopathological parameters, cancer tumor node metastasis stage, immunohistochemical staining method, antibody source, percentage rate of expression, and follow-up. Data for study characteristics and clinical responses were summarized and the data turned into table format. Heterogeneity of investigation was evaluated to determine whether the data of the various studies could be analyzed for a meta-analysis.

For the methodological evaluation of the studies, three investigators read through each publication independently and assessed and scored them according to REMARK guidelines and the ELCWP quality scale (Steels et al., 2001; McShane et al., 2005). Any discrepancies or disagreements were discussed, and if consensus could not be achieved, a third reviewer resolved the issue.

\section{Statistical analysis}

Analysis was conducted using the STATA 12 (StataCorp LP, College Station, TX, USA) and Review Manager 5.2 (Cochrane Collaboration, Oxford, UK). Heterogeneity among studies was evaluated by $\mathrm{I}^{2}$ inconsistency test and $\chi^{2}$-based Cochran's Q statistic test (Higgins et al., 2003). When heterogeneity was not an issue ( $\mathrm{I}^{2}<50 \%$ or $\left.\mathrm{p} \geq 0.05\right)$, a fixed-effect model was used to calculate parameters. If there was substantial heterogeneity $\left(\mathrm{I}^{2} \geq 50 \%\right.$ or $\left.p<0.05\right)$, a random-effects model was used to pool data and attempted to identify potential sources of heterogeneity based on subgroup analyses. The pooled odds ratio (OR) was estimated for the association between DLC-1 expression and clinicopathological features. All reported $p$ values were two-sided and $p<0.05$ was considered statistically significant.

The possibility of publication bias was assessed using the Begg test and visual inspection of a funnel plot (Begg and Mazumdar, 1994; Egger et al., 1997) We also performed the Duval and Tweedie nonparametric "trim and fill" procedure to further assess the possible effect of publication bias in our meta-analysis (Higgins and Thompson, 2002).This method considers the possibility of hypothetical "missing" studies that might exist, imputes their ORs, and recalculates a pooled OR that incorporates the hypothetical missing studies as though they actually existed.

\section{Results}

\section{Eligible studies and characteristics}

Two hundred and eighty-eight publications were identified by the search method as described above. Two hundred and sixty-nine of those were excluded due to being non-original articles (review), or studies irrelevant to the current analysis. Eventually, based on the above inclusion and exclusion criteria, nineteen studies were included in this meta-analysis, as shown in Figure 1. total of 1815 tumor patients were enrolled. Their basic characteristics are summarized in Table 1.

The correlation of CXCR4 expression with clinicopathological features

\section{Increased DLC-1 expression in cancers}

We first determined whether DLC-1 expression is significantly higher in cancers than in normal tissues. The pooled OR from nineteen studies including 1182 cancers and 633 normal tissues is shown in Figure 2. There was no significant heterogeneity $\left(\mathrm{I}^{2}=0.0 \%, p=0.782\right)$, and the pooled OR was performed using a fixed model $(\mathrm{OR}=0.441$, 95\% $\mathrm{CI}=0.374-0.520, p=0.000$ ), which indicated that DLC-1 expression is significantly lower in cancers than in the normal tissues.

\section{The role of DLC-1 expression in cancer progression}

We then analyzed 810 cancer patients pooled from thirteen studies to assess whether DLC-1 expression in cancers was associated with advanced stage. As shown in Figure 2, aberrant DLC-1 expression was significantly lower in advanced cancers (stages III and IV) than in earlystage cancers ( stages I and II) $(\mathrm{OR}=0.435,95 \% \mathrm{CI}=0.332$ $0.570, p=0.000)$ by fixed model $\left(\mathrm{I}^{2}=0.0 \%, p=0.993\right)$. In addition, as shown in Figure Supplement 1, aberrant DLC-1 expression was not significantly lower in poorly and moderately differentiated cancers than in highly

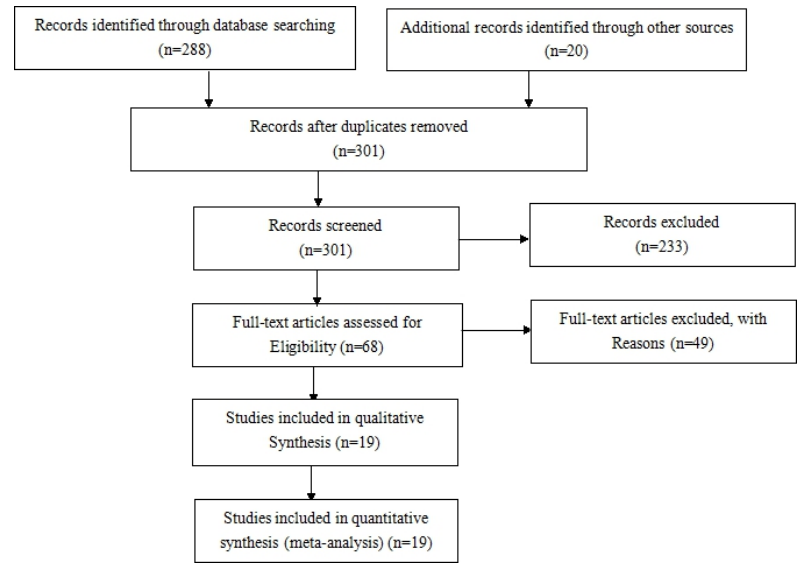

Figure 1. Flow Chart of Study Selection 


\begin{tabular}{|c|c|c|c|c|c|c|c|c|c|c|c|c|c|c|c|c|c|c|c|}
\hline 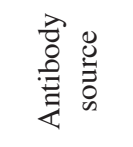 & 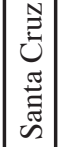 & 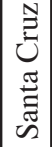 & 分 & 孚 & 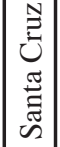 & 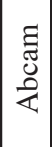 & 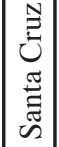 & 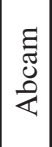 & 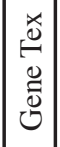 & 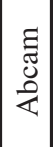 & 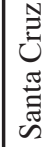 & 吕 & 参 & 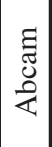 & $\frac{q}{z}$ & 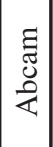 & 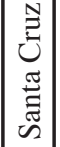 & 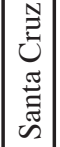 & 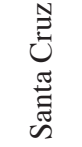 \\
\hline 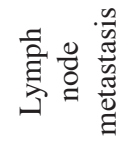 & 参 & 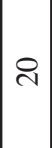 & $\frac{\alpha}{z}$ & $\frac{a}{z}$ & $m$ & $\bar{m}$ & オ & in & $\hat{\imath}$ & $\stackrel{\leftrightarrow}{\sim}$ & $\hat{m}$ & 孚 & $\mid \frac{a}{z}$ & तิ & $a$ & 2 & $\stackrel{+}{m}$ & $\frac{\kappa}{z}$ & 学 \\
\hline 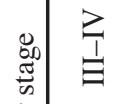 & 孚 & ৪্ল & $\ddot{\imath}$ & 孚 & $f$ & 8 & ร & $\overline{5}$ & ৪্ল & প্ল & I & $=$ & $\mid \frac{a}{z}$ & $=$ & $\approx$ & సి & $\dot{n}$ & $\frac{\kappa}{z}$ & $\vec{\lambda}$ \\
\hline 声 & 参 & $\stackrel{\infty}{-}$ & $\simeq$ & 孚 & $\tilde{m}$ & $\stackrel{0}{-}$ & $\stackrel{\sim}{0}$ & $\hat{n}$ & $\cong$ & $\bar{m}$ & లি & 의 & 孚 & $\vec{\imath}$ & $\stackrel{\infty}{\sim}$ & $\tilde{\curvearrowright}$ & $\stackrel{\infty}{+}$ & 孚 & $\bar{F}$ \\
\hline $\begin{array}{l}\tilde{z} \\
0 \\
\vec{E} \\
\sum\end{array}$ & 胥 & 五 & 总 & $\begin{array}{l}\frac{\alpha}{0} \\
0 \\
\frac{1}{\alpha} \\
\frac{1}{\alpha}\end{array}$ & 兽 & 兽 & 曽 & 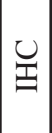 & 诖 & 兽 & 兽 & $\begin{array}{l}\frac{\alpha}{0} \\
0 \\
\frac{1}{\alpha} \\
\frac{1}{\alpha}\end{array}$ & 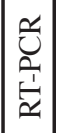 & 兽 & 呈 & 导 & 呈 & 呈 & 岕悹 \\
\hline 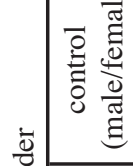 & 参 & $\frac{0}{n}$ & 泀 & $\stackrel{\overbrace{}}{\mathrm{I}}$ & 告 & ฮิ & $\mid \begin{array}{c}\tilde{a} \\
\text { ठิ }\end{array}$ & $\frac{r}{z}$ & $\frac{\tilde{z}}{\mathrm{z}}$ & 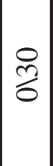 & $\frac{8}{1}$ & $\stackrel{o}{0}$ & $\stackrel{\infty}{=}$ & $\frac{r}{z}$ & $\frac{a}{z}$ & 孚 & वิ & $\frac{a}{z}$ & 号 \\
\hline 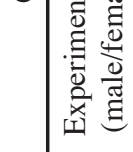 & 参 & 胥 & 产 & 言 & $\begin{array}{l}n \\
\frac{2}{\sigma}\end{array}$ & $\begin{array}{l}0 \\
\frac{0}{6}\end{array}$ & 点 & 售 & 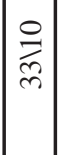 & $\overrightarrow{0}$ & है & $\underset{\sim}{\stackrel{Q}{\sim}}$ & 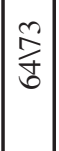 & 兽 & $\frac{2}{n}$ & $\begin{array}{l}0 \\
\vdots \\
0 \\
\end{array}$ & $\begin{array}{l}0 \\
0 \\
\sigma\end{array}$ & $\frac{\mathfrak{z}}{\mathrm{z}}$ & $\underset{\overrightarrow{7}}{\vec{\gamma}}$ \\
\hline $\begin{array}{l}\overrightarrow{0} \\
\stackrel{0}{0}\end{array}$ & 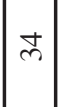 & 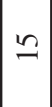 & \pm & 10 & 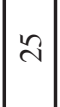 & సి & สิ & సి & ¿ి| & ৪্লি & $\mathcal{F}$ & ○ & $\stackrel{\infty}{+}$ & q & 8 & $\stackrel{\infty}{+}$ & ते & $\infty$ & $\widetilde{\sigma}$ \\
\hline 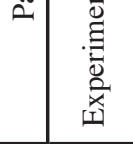 & $\underset{\sim}{\infty}$ & $\stackrel{\infty}{+}$ & $m$ & $F$ & $\because$ & 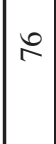 & $\widehat{\sigma}$ & 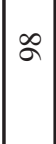 & $\mathscr{F}$ & $\overline{6}$ & $\tilde{n}$ & $\bar{\imath}$ & $\widehat{2}$ & $q$ & 8 & $\stackrel{\infty}{+}$ & $\delta$ & $\infty$ & $\widetilde{\sigma}$ \\
\hline $\overrightarrow{\overline{0}}$ & 㢺 & 离 & 节 & 鴗 & 离 & 茎 & 竺 & 刭 & 竎 & 节 & 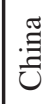 & 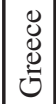 & 节 & 㞼 & 节 & 莞 & 親 & 昙 & 苞 \\
\hline$\stackrel{\bar{d}}{D}$ & 离 & $\stackrel{\sim}{\stackrel{7}{7}}$ & $\frac{\text { d }}{4}$ & : & 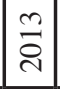 & $\overline{\vec{c}}$ & \begin{tabular}{l}
$\sim$ \\
\multirow{2}{*}{} \\
\end{tabular} & 을 & $\frac{2}{\overrightarrow{2}}$ & $\vec{\nabla}$ & 을 & : & 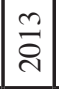 & 离 & 年 & 离 & $\begin{array}{l}\Delta \\
\\
\end{array}$ & \begin{tabular}{l}
$m$ \\
\multirow{2}{*}{} \\
\end{tabular} & ஓे \\
\hline 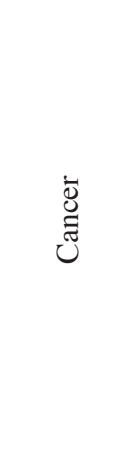 & 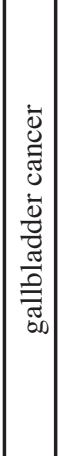 & 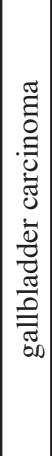 & 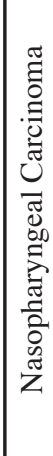 & 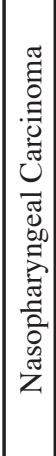 & 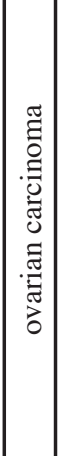 & 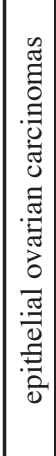 & 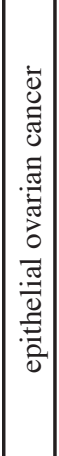 & 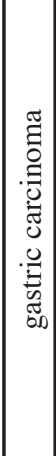 & 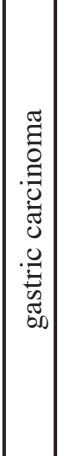 & 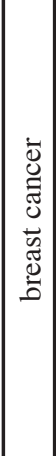 & 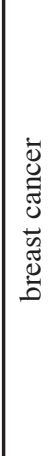 & 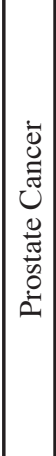 & 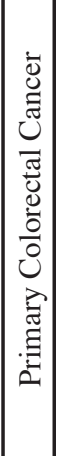 & 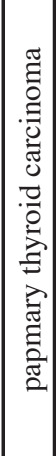 & 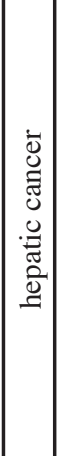 & 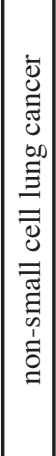 & 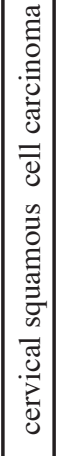 & 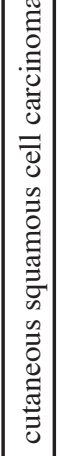 & 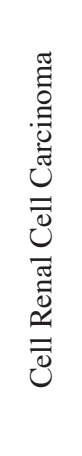 \\
\hline 胥 & 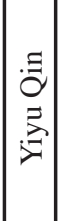 & 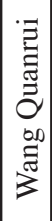 & 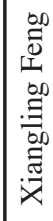 & $\begin{array}{l}00 \\
\overline{0} \\
2 \\
\tilde{\Xi} \\
0 \\
0\end{array}$ & 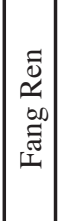 & 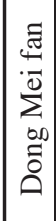 & 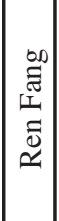 & 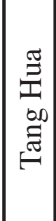 & 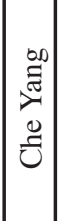 & 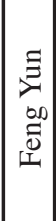 & 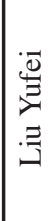 & 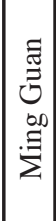 & 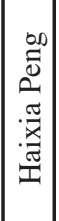 & | & 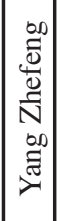 & 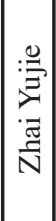 & 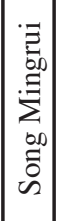 & 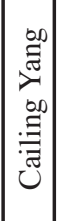 & $\begin{array}{l}\text { 怘 } \\
\text { Ñ } \\
\text { 品 }\end{array}$ \\
\hline
\end{tabular}




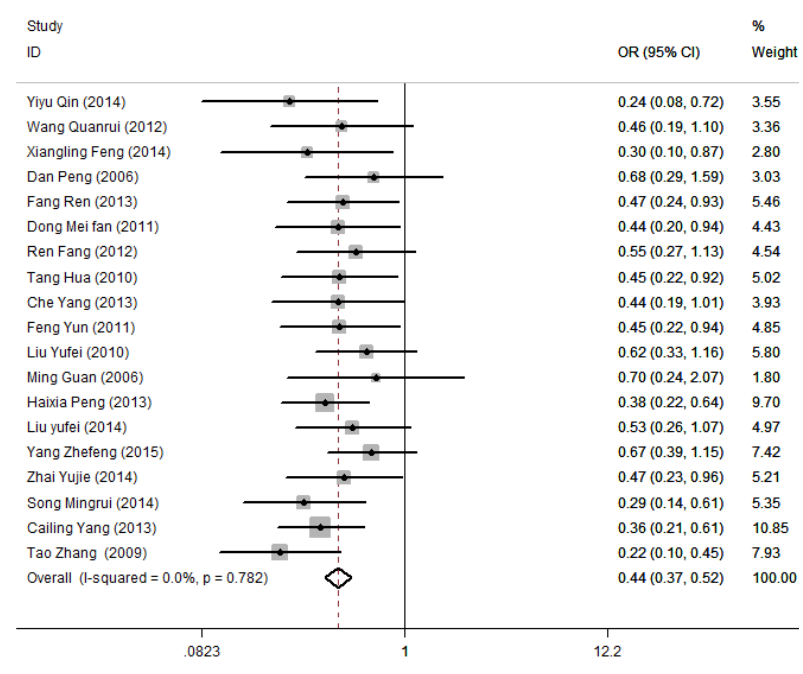

Figure 2. The Pooled OR of DLC-1 Expression from Nineteen Studies Including 1182 Cancers and 633 Normal Tissues $(O R=0.916,95 \%$ CI $=0.552-1.519$, $p=\mathbf{0 . 7 3 4}$ ). Abbreviations: OR, odds ratio; CI, confidence interval; DLC-1, deleted in liver cancer-1

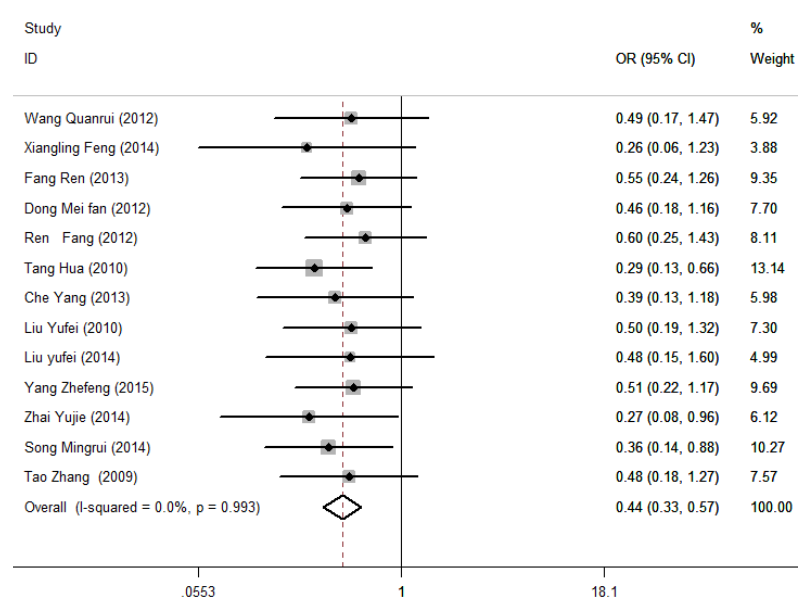

Figure 3. DLC-1 expression was Significantly Lower in Advanced Cancers (stages III and IV) than in EarlyStage Cancers (Stages I and II) Pooled from Thirteen Studies Including 810 Cancer Patients. Abbreviations: OR, odds ratio; CI, confidence interval; DLC-1, deleted in liver cancer-1

differentiated cancers $(\mathrm{OR}=0.916,95 \% \mathrm{CI}=0.552-1.519$, $p=0.734)$. These results suggest that DLC -1 expression may not associate with tumor's differentiated status, but may play an important role in cancer progression and development.

The role of DLC-1 expression in metastatic cancers

We then analyzed 744 cancer patients pooled from twelve studies to assess whether DLC-1expression in cancers was associated with lymphoma metastatic status. As shown in Figure 3, there was no significant heterogeneity $\left(\mathrm{I}^{2}=0.0 \%, p=0.910\right)$ among studies and the pooled OR was performed using a fixed model. Aberrant DLC-1 expression was significantly lower in metastatic cancers than in nonmetastatic cancers $(\mathrm{OR}=0.432,95 \%$ $\mathrm{CI}=0.323-0.579, \mathrm{P}=0.000)$. The result suggests that DLC1 expression is strongly correlated with metastatic status in cancer patients.

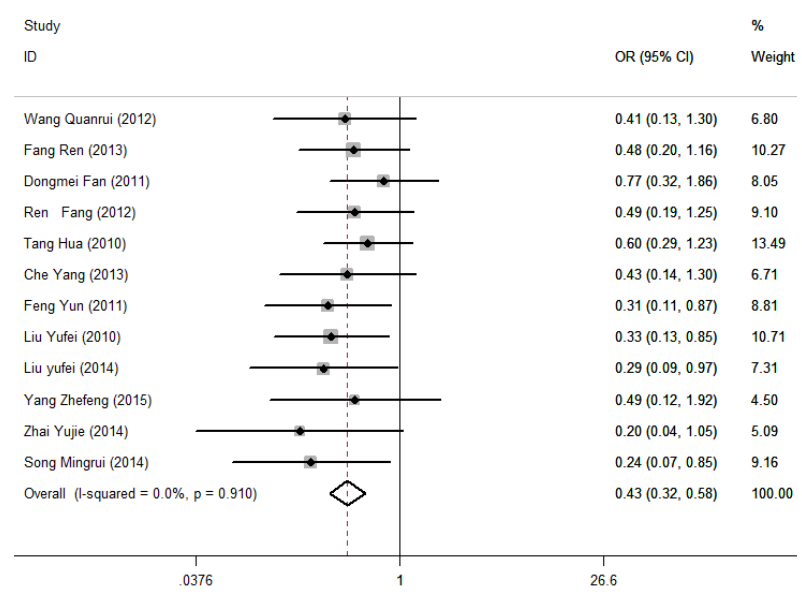

Figure 4. DLC-1 expression was Significantly Lower in Metastatic Cancers than in Nonmetastatic Cancers Pooled from Twelve Studies Including 744 Cancer Patients $(\mathrm{OR}=\mathbf{0 . 4 3 2}, 95 \% \mathrm{CI}=\mathbf{0 . 3 2 3}-\mathbf{0 . 5 7 9}, \mathrm{P}=\mathbf{0 . 0 0 0})$. Abbreviations: OR, odds ratio; $\mathrm{CI}$, confidence interval; DLC-1 deleted in liver cancer-1
A

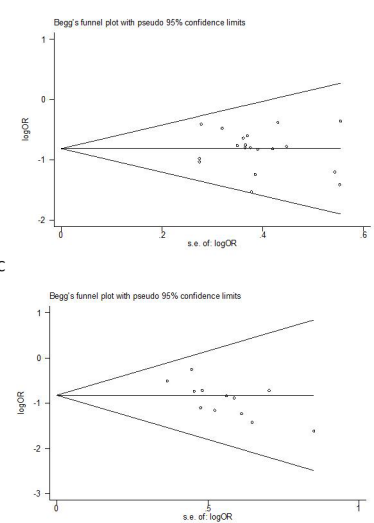

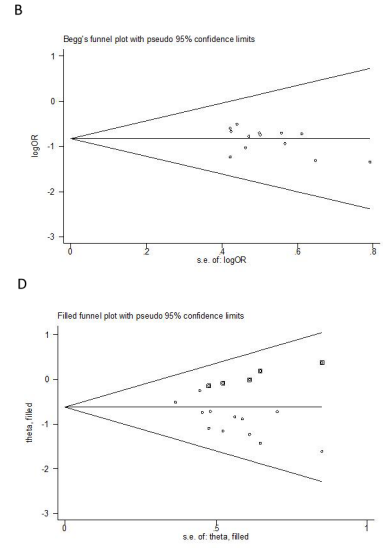

Figure 5. The Funnel Plots of Publication Biases in the Meta-Analysis of DLC-1 Expression and Clinicopathological Features. (A) The funnel plot from nineteen studies compared cancers and the normal tissues, there is no significant publication bias ( $p=0.441$ ). (B) The funnel plot from thirteen ten studies in determing DLC-1 expression for different stages of cancers, there is no significant publication bias $(p=0.161)$. (C) The funnel plot from twelve studies determined the relationship between DLC-1 expression and metastatic status in cancers $(p=0.011)$. (D) The funnel plot with Trim and Fill from seventeen studies determined the relationship between DLC-1 expression and metastatic status in cancers

\section{Sensitivity analyses and publication bias}

A sensitivity analysis, in which one study was removed at a time, was conducted to assess the stability of the results. The pooled ORs were not significantly changed, indicating the stability of our analyses. Two funnel plots were largely symmetric (Figure 4A-B) suggesting there were no publication biases in the meta-analysis of DLC-1 expression in cancers $(p=0.441)$ and DLC- 1 expression with cancer progression $(p=0.161)$. Visual inspection of the Begg funnel plot revealed asymmetry in the analysis of DLC-1 expression with cancer metastasis (Figure 4C), and the Begg test was also statistically significant $(p=0.011)$. This indicated the possibility of publication bias. Because of this, we undertook a sensitivity analysis using the trim and fill method (Duval and Tweedie, 2000), which 


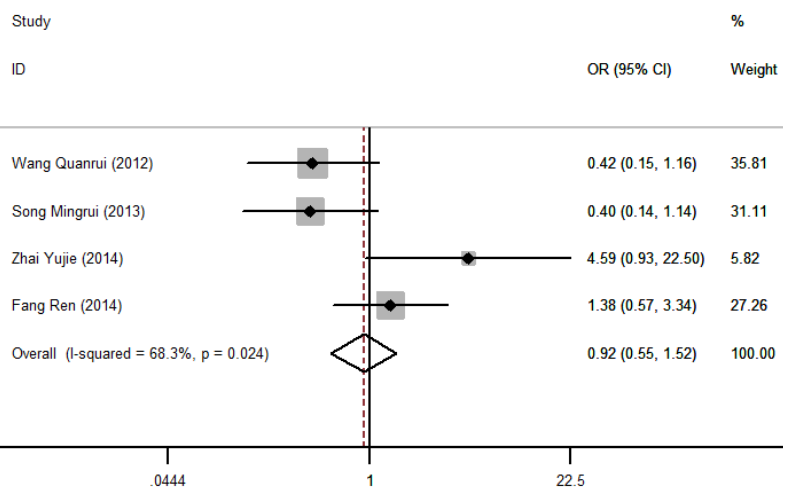

Figure Supplement 1. DLC-1 Expression was not Significantly Lower in Poorly and Moderately Differentiated Cancers than in Highly Differentiated Cancers $(\mathrm{OR}=\mathbf{0 . 9 1 6 , 9 5 \%} \mathrm{CI}=\mathbf{0 . 5 5 2} \boldsymbol{- 1 . 5 1 9}, p=0.734)$. Abbreviations: OR, odds ratio; CI, confidence interval; DLC-1, deleted in liver cancer-1

conservatively imputes hypothetical negative unpublished studies to mirror the positive studies that cause funnel plot asymmetry. The imputed studies produce a symmetrical funnel plot (Figure 4D). The pooled analysis incorporating the hypothetical studies continued to show a statistically significant association between DLC-1 expression and cancer metastasis $(\mathrm{OR}=0.537,95 \% \mathrm{CI}=0.416-0.693$, $\mathrm{P}=0.000)$

\section{Discussion}

To date, there have been some studies describing the precise expression and prognostic impact of DLC-1 in cancers. However, the roles and clinical significance of DLC-1 expression in cancers have not been thoroughly investigated. We conducted the meta-analysis to determine the correlation between DLC-1 expression and clinicopathological characteristics in cancers. Analyses of the pooled data showed that cancers had a significantly lower DLC-1expression than normal tissues $(\mathrm{OR}=0.435$, $95 \% \mathrm{CI}=0.332-0.570, p=0.000)$. Recent studies have shown that down-regulation or inactivation of the DLC-1 gene during tumor development appears to be primarily due to aberrant methylation at the gene promoter. In an early study, the promoter methylation was proved to major responsible for down-regulation of DLC-1 by screening several cell lines derived from HCC, prostate tumors and colon (Yuan et al., 2003). Then the analysis of 73 surgical samples of HCC showed that the level of DLC1methylation in highly invasive HCC was significantly higher than in low invasion cases (Liu, 2008). A recent methylation analysis of 68 patients with pancreatic ductal adenocarcinoma showed that DLC-1 hypermethylation strongly correlated with lymph node metastasis (Xue et al., 2013). Promoter methylation and $\mathrm{LOH}$ result in DLC-1 inactivation in nasopharyngeal tumors (Feng et al., 2014). Still, more studies are needed to reveal the relationship betweeen DLC-1 expression and promoter methylation, and the affect of promoter methylation on clinicopathological features.

DLC-1 also played an important role in cancer progress and metastasis. This meta-analysis pooled the data and showed that aberrant DLC-1 expression was significantly lower in metastatic cancers than in nonmetastatic cancers $(\mathrm{OR}=0.432,95 \% \mathrm{CI}=0.323-0.579, \mathrm{P}=0.000)$ and in advanced cancers (stages III and IV) than in early-stage cancers (stages I and II) $(\mathrm{OR}=0.435,95 \% \mathrm{CI}=0.332$ - $0.570, p=0.000)$,while aberrant DLC-1 expression was not significantly lower in poorly and moderately differentiated cancers than in highly differentiated cancers ( $\mathrm{OR}=0.916,95 \% \mathrm{CI}=0.552-1.519, p=0.734)$. The results suggest that DLC-1 expression play an important role in cancer progression and metastasis even not in cancer differentiation. Because of limited studies involve in cancer differentiation analysis, the differentiation result is not certain and need more new evidence to prove it. The mechanism that DLC-1 expression inhibits cancer progression was focused on DLC-1 interaction proteins. Members of the tensin family of focal adhesion proteins were identified the first DLC-1 binding partners and the impact of this interaction has been examined in HCC, NSCLC and breast carcinoma cells (Yam et al., 2006; Qian et al., 2007). Other proteins, like phosphatase and tensin homolog (PTEN), p120Ras-GAP (RASA1) and S100A10 are also studied in several cancers and targeting prooncogenic proteins activated by DLC-1 downregulation was thought to be therapeutically effective for the suppression of cancer progression and metastasis (Heering et al., 2009; Yang et al., 2009; Yang et al., 2011).

Consistent results were shown in sensitivity analyses, and no evidence of heterogeneity was found. This metaanalysis met publication bias issues when analyze the association between DLC-1 expression and cancer metastasis. We undertook a sensitivity analysis using the trim and fill method to remove publication bias and the pooled analysis after adjusted continued to show a statistically significant association between DLC-1 expression and cancer metastasis. This study has several potential limitations. First, the possibility of information and selection biases as well as unidentified confounders could not be completely excluded because all of the included studies were observational. Second, the searching strategy was restricted to articles published in English and Chinese. Articles with potentially high-quality data that were published in other languages were not included. Third, the samples and studies were limited by a presence of heterogeneity between other studies.

In conclusion, Our meta-analysis showed that DLC1 expression was significantly lower in cancers than in normal tissues. The aberrant DLC-1 expression plays an important role in cancer carcinogenesis and metastasis. Thus, it is safe to say that the remarkable potential of DLC-1 could serve as a prognostic biomarker for cancer patients. Further large-scale studies, especially multicenter, could serve well-matched cohort will provide more insight into the role of DLC-1 in the prognosis and clinical implementation of cancer patients.

\section{Acknowledgements}

This study was supported by construct program of the key discipline in hunan province and the grant from Foundation of the Changsha Medical University (2013) 


\section{References}

Begg CB, Mazumdar M (1994). Operating characteristics of a rank correlation test for publication bias. Biometrics, 50, 1088-101.

Duval S,Tweedie R (2000). Trim and fill: A simple funnel-plotbased method of testing and adjusting for publication bias in meta-analysis. Biometrics, 56, 455-63.

Egger M, Davey Smith G, Schneider M, et al (1997). Bias in metaanalysis detected by a simple, graphical test. BMJ, 315, 629-34.

Etienne-Manneville S, Hall A (2002). Rho GTPases in cell biology. Nature, 420, 629-35.

Fan DM, Shi HR (2011). Pilot study: alteration of deleted in liver cancer1 and phosphorylated focal adhesion kinase Y397 cytoplasmic expression and the prognostic value in advanced epithelial ovarian carcinoma. Int J Mol Sci, 12, 8489-501.

Fang R, Huirong S, Zhimin C, et al (2012). Protein expression of DLC-1, FAK and Crk-associated $130 \mathrm{kDaP}$ in epithelial ovarian cancer and their significance. Modern Oncol, 21, $152-5$.

Feng X, Ren C, Zhou W, et al (2014). Promoter hypermethylation along with $\mathrm{LOH}$, but not mutation, contributes to inactivation of DLC-1 in nasopharyngeal carcinoma. Mol Carcinog, 53, 858-70.

Guan M, Zhou X, Soulitzis N, et al (2006). Aberrant methylation and deacetylation of deleted in liver cancer-1 gene in prostate cancer: potential clinical applications. Clin Cancer Res, 12, 1412-9.

Heering J, Erlmann P, Olayioye MA (2009). Simultaneous loss of the DLC1 and PTEN tumor suppressors enhances breast cancer cell migration. Exp Cell Res, 315, 2505-14.

Higgins JP, Thompson SG (2002). Quantifying heterogeneity in a meta-analysis. Stat Med, 21, 1539-58.

Higgins JP, Thompson SG, Deeks JJ, et al (2003). Measuring inconsistency in meta-analyses. $B M J, 327,557-60$.

Homma Y, Emori Y (1995). A dual functional signal mediator showing RhoGAP and phospholipase C-delta stimulating activities. EMBO J, 14, 286-91.

Hua T, Xiaojun Y, Tie Z, et al (2010). The manifestation of DLC1 proteins in gastric carcinoma and its clinic-pathological significance. Anhui Med J, 31, 1328-31.

Liu JB ZS, Shi MX, Shao BF, Zhang YX (2008). Relationship between methylation status of DLC-1 gene and metastasis of hepatocellular carcinoma. World Chinese J Digestol, 11, 1237-40.

McShane LM, Altman DG, Sauerbrei W, et al (2005). Reporting recommendations for tumor marker prognostic studies (REMARK). J Natl Cancer Inst, 97, 1180-4.

Mingrui S, Hui C, Xia-yun Z, et al (2014). Expression of DLC1 and cycfin D1 and their clinical significance in cervical squamous cell carcinoma. Chinese J Diagnostic Pathol, 21, 633-6.

Moon SY, Zheng Y (2003). Rho GTPase-activating proteins in cell regulation. Trends Cell Biol, 13, 13-22.

Peng D, Ren CP, Yi HM, et al (2006). Genetic and epigenetic alterations of DLC-1, a candidate tumor suppressor gene, in nasopharyngeal carcinoma. Acta Biochim Biophys Sin (Shanghai), 38, 349-55.

Peng H, Long F, Wu Z, et al (2013). Downregulation of DLC-1 gene by promoter methylation during primary colorectal cancer progression. Biomed Res Int, 2013, 181384.

Qian X, Li G, Asmussen HK, et al (2007). Oncogenic inhibition by a deleted in liver cancer gene requires cooperation between tensin binding and Rho-specific GTPase-activating protein activities. Proc Natl Acad Sci U S A, 104, 9012-7.

Qin Y, Chu B, Gong W, et al (2014). Inhibitory effects of deleted in liver cancer 1 gene on gallbladder cancer growth through induction of cell cycle arrest and apoptosis. J Gastroenterol Hepatol, 29, 964-72.

Quanrui W, Zhimei C (2012). Expression and signiiieance of DLC-
1 protein in gallbladder carcinoma.J Hepatopancreatobiliarv Surg, 24, 214-6.

Ren F, Shi H, Zhang G, et al (2013). Expression of deleted in liver cancer 1 and plasminogen activator inhibitor 1 protein in ovarian carcinoma and their clinical significance. J Exp Clin Cancer Res, 32, 60.

Seng TJ, Low JS, Li H, et al (2007). The major 8p22 tumor suppressor DLC1 is frequently silenced by methylation in both endemic and sporadic nasopharyngeal, esophageal, and cervical carcinomas, and inhibits tumor cell colony formation. Oncogene, 26, 934-44.

Steels E, Paesmans M, Berghmans T, et al (2001). Role of p53 as a prognostic factor for survival in lung cancer: a systematic review of the literature with a meta-analysis. Eur Respir $J$, 18, 705-19.

Tcherkezian J, Lamarche-Vane N (2007). Current knowledge of the large RhoGAP family of proteins. Biol Cell, 99, 67-86.

Wang Y, Lei R, Zhuang X, et al (2014). DLC1-dependent parathyroid hormone-like hormone inhibition suppresses breast cancer bone metastasis. J Clin Invest, 124, 1646-59.

Guan CN, Zhang PW, Lou HQ, et al (2012). DLC-1 expression levels in breast cancer assessed by qRT- PCR are negatively associated with malignancy. Asian Pac J Cancer Prev, 13, 1231-33.

Xue YZ, Wu TL, Wu YM, et al (2013). DLC-1 is a candidate biomarker methylated and down-regulated in pancreatic ductal adenocarcinoma. Tumour Biol, 34, 2857-61.

Yam JW, Ko FC, Chan CY, et al (2006). Interaction of deleted in liver cancer 1 with tensin 2 in caveolae and implications in tumor suppression. Cancer Res, 66, 8367-72.

Yang C, Tiankang G, Yuntao M, et al (2013a). Expression and clinical significance of DLC-1 and MMP-9 in gastric carcinoma. J Ningxia Med University, 35, 872-6.

Yang C, Wu D, Jia J, et al (2013b). DLC1 as a regulator of proliferation, invasion, cell cycle, and apoptosis in cutaneous squamous cell carcinoma. Tumour Biol, 34, 2633-43.

Yang X, Popescu NC, Zimonjic DB (2011). DLC1 interaction with S100A10 mediates inhibition of in vitro cell invasion and tumorigenicity of lung cancer cells through a RhoGAPindependent mechanism. Cancer Res, 71, 2916-25.

Yang XY, Guan M, Vigil D, et al (2009). p120Ras-GAP binds the DLC1 Rho-GAP tumor suppressor protein and inhibits its RhoA GTPase and growth-suppressing activities. Oncogene, 28, 1401-9.

Yuan BZ, Durkin ME, Popescu NC (2003). Promoter hypermethylation of DLC-1, a candidate tumor suppressor gene, in several common human cancers. Cancer Genet Cytogenet, 140, 113-7.

Yuan BZ, Miller MJ, Keck CL, et al (1998). Cloning, characterization, and chromosomal localization of a gene frequently deleted in human liver cancer (DLC-1) homologous to rat RhoGAP. Cancer Res, 58, 2196-9.

Yufei L, Shengrong S, Chuan C, et al (2014). Protein expression and promoter methylation of deleted in liver cancer-1 gene in papilarry thyroid carcinoma. Chin J Exp Surg, 31, 152-5.

Yufei L, Zhaoxiang Z, Lin H, et al (2010). Expression of DLC1 protein and DLC1-mRNA in breast carcinoma and their clinical significance. Chinese J Clin Oncol, 37, 866-9.

Yujie Z, Qingshuai F, Fangling N, et al (2014). Expressions and clinical significance of DLC1 and ROCK1 in non small cell lung cancer. J Int Oncol, 41, 688-91.

Yun F, Huixing Z, Junhong L, et al (2011). Expression of deleted in liver cancer 1 and phosphorelated focal adhesion kinase in breast cancer. $J$ South Med Univ, 31, 1448-551.

Zhang T, Zheng J, Liu C, et al (2009). Expression of DLC-1 in clear cell renal cell carcinoma: prognostic significance for progression and metastasis. Urol Int, 82, 380-7.

Zhefeng Y, Xisheng Y, Bin D, et al (2015). Clinical significance of DLC-1 expression in hepatic cancer. Modern Oncol, 23, 644-6.1 